\title{
IV INHALTSVERZEICHNIS
}

\begin{tabular}{|c|c|c|}
\hline CIL III & & CIL IV \\
\hline 107 I 6 & 509 & 549 \\
\hline 10717 & 798 & 567 \\
\hline I 2067 & $2 \mathrm{I} 4 \mathrm{~b}$ & $58 \mathrm{I}$ \\
\hline I 2068 & $2 \mathrm{I}_{4} \mathrm{C}$ & 597 \\
\hline I 2069 & 2 I $4 a$ & 652 \\
\hline CIL IV & & 653 \\
\hline 7 & I 20 & 659 \\
\hline I 7 & 122 & 669 \\
\hline 20 & 124 & 677 \\
\hline $2 \mathrm{I}$ & I 2 I & 733 \\
\hline 26 & 125 & 760 \\
\hline 27 & 159 & 768 \\
\hline 29 & I9I & $79 \mathrm{If}$. \\
\hline 45 & 126 & 794 \\
\hline 60 & I I 2 & $806 \mathrm{f}$. \\
\hline $6 I$ & $15^{8}$ & 812 \\
\hline 64 & 432 & $8 \mathrm{I}_{3}$ \\
\hline 67 & I 23 & 826 \\
\hline 88 & 711 & 840 \\
\hline 89 & 629 & 844 \\
\hline 99 & 130 & $85^{8}$ \\
\hline 103 & 129 & 864 \\
\hline I I I & I64 & 879 \\
\hline I 20 & I 68 & 882 \\
\hline 128 & I 66 & 918 \\
\hline 138 & 437 & 920 \\
\hline I 48 & 3 IO & 932 \\
\hline 175 & I 88 & 933 \\
\hline 187 & I 53 & $95^{\circ}$ \\
\hline 202 & I 40 & $994 \mathrm{f}$. \\
\hline $22 \mathrm{I}$ & 170 & IOI I \\
\hline 222 & 156 & I059 \\
\hline 259 & 99 & I069a \\
\hline 275 & 172 & Jo74 \\
\hline 309 & 177 & 1084 \\
\hline 346 & 167 & 1085 \\
\hline 362 & $15 I$ & 1093 \\
\hline 387 & 165 & I094 \\
\hline 423 & 135 & Iog6 \\
\hline 427 & 190 & $1096 \mathrm{a}$ \\
\hline 429 & 179 & I I I I \\
\hline 470 & 173 & I I 8 \\
\hline 528 & 96 & I I 22 \\
\hline 538 & 263 & I I 36 \\
\hline $54^{8}$ & 202 & \\
\hline
\end{tabular}

\begin{tabular}{|c|c|c|}
\hline 484 & $\underset{1176}{\text { CIL IV }}$ & 8 \\
\hline 187 & I 177 & 244 \\
\hline 169 & I 178 & I 17 \\
\hline I 57 & I 79 & 243 \\
\hline 579 & 1180 & 245 \\
\hline 713 & I I 90 & 246 \\
\hline $\begin{array}{r}537 \\
84\end{array}$ & $\begin{array}{l}\text { I } 2 \text { I I f. } \\
\text { I } 226\end{array}$ & $\begin{array}{l}345 \\
131\end{array}$ \\
\hline I 86 & 1227 & 14 \\
\hline 10 & $123^{\circ}$ & 618 \\
\hline 503 & I 234 & 510 \\
\hline 137 & 1237 & 476 \\
\hline I 39 & I 239 & 403 \\
\hline 716 & I 24 I & 53 I \\
\hline 439 & I 256 & 576 \\
\hline 20 & I $26 \mathrm{I}$ & 617 \\
\hline 704 & I 266 & 209 \\
\hline I $8 \mathrm{I}$ & I 277 & 333 \\
\hline I34 & 1279 & IOI \\
\hline I5 & I 283 & 578 \\
\hline 136 & I 289 & 339 \\
\hline 184 & I 29I & 631 \\
\hline 472 & I 292 & 632 \\
\hline I 2 & I 293 & 297 \\
\hline 763 & I 294 & 309 \\
\hline 197 & I 309 & 284 \\
\hline 146 & I 3 I 4 & 495 \\
\hline I 45 & $132 \mathrm{I}$ & 707 \\
\hline 709 & I 324 & 804 \\
\hline 141 & I 326 & 569 \\
\hline I7 I & 1329 & 298 \\
\hline $13^{8}$ & I33 I & $649 a$ \\
\hline 212 & 1337 & 512 \\
\hline 94 & I347 & 560 \\
\hline 83 & 1353 & 349 \\
\hline 3 I I & I 354 & 44 \\
\hline 519 & I 355 & 374 \\
\hline 285 & I357 & $57 \mathrm{I}$ \\
\hline 444 & I 363 & 656 \\
\hline 443 & I 364 & 823 \\
\hline 300 & I $388 \mathrm{a}$ & $66 I$ \\
\hline 567 & $13^{89}$ & 657 \\
\hline I 54 & 1391 & 625 \\
\hline 435 & I 392 & 425 \\
\hline 594 & I 393 & 426 \\
\hline
\end{tabular}


CIL IV

I396

I397

1398

$1410 \mathrm{f}$.

I $42 \mathrm{I}$

1422

1425

I 427

I 435

I 44 I

I 446

1449

I 454

1463

1474

1477

I 507

I5 I 2

I5 6

1517

1520

I 527

I 529

1536

I $53^{8}$

I 544

I 545

I 547 e

$155^{2}$

1553

I 555

I 560

I 564

I $570 \mathrm{f}$.

I574

I 589

1590

I 592

I 593

1595

I 597

I604

I 607

I6I 8

1621

1625

I626

I 635

I 644

I 645

1649

I 650

\begin{tabular}{r|l} 
& CIL IV \\
37 & 1653 \\
492 & 1654 \\
530 & 1655 \\
515 & 1658 \\
261 & $\mathrm{~J} 662$
\end{tabular}

262 I 664

649 I678

660 I679

$3 1 9 \longdiv { 1 6 8 4 }$

$504 \quad I 688$

$712 \quad 1690$

$733 \quad \mathbf{1} 698$

6 I 700

364 I 17 I

278 I 712

558 I 713

$427 \quad 1714$

562 I 731

615,1751

624 I754

$785 \quad 1763$

\begin{tabular}{l|l}
789 & 1768
\end{tabular}

$653 \quad 1773$

25 I 780

$813178 \mathrm{I}$

IO9 1783

93 I 787

348 I 816

I07 1819

1051820

$108 \quad 1824$

418 I 825

$334 \quad 1826$

$438 \quad 1830$

486 I 83 I

\begin{tabular}{l|l}
2 & 1837
\end{tabular}

$336 \quad 1838$

$3 3 5 \longdiv { 1 8 3 9 }$

480 I 84 I

304 I 842

\begin{tabular}{l|l}
429 & 1847
\end{tabular}

$4 9 6 \longdiv { 1 8 4 9 }$

513 r $849 a$

$770 \quad \mathrm{I} 852$

$36 \mathrm{I} \quad \mathrm{I} 854$

$26 \quad 1856$

\begin{tabular}{l|l} 
I6 & I 858
\end{tabular}

$678 \div 1860$

I $7 \quad 1863$

$600 \times 1864$

$592 \quad 1870$

7051877 \begin{tabular}{l|ll} 
& CIL IV & \\
283 & I 880 & $64 I$ \\
81 I & I 881 & $49 I$ \\
545 & I 882 & 689 \\
I 99 & I 883 & 583
\end{tabular}

708 I 885 I06

28I I $886 \quad$ I94

$\begin{array}{lll}410 & \text { I } 890 & 358\end{array}$

34 I 89 I $\quad 482$

$474 \quad I 893 \quad 805$

$774 \quad 1894 \quad 788$

$555 \quad 1895 \quad 803$

679 I $896 \quad 782$

\begin{tabular}{l|l}
666 & 1898 \\
297
\end{tabular}

\begin{tabular}{l|ll}
207 & 1899 & $\mathbf{8 2 8}$
\end{tabular}

\begin{tabular}{l|ll}
417 & 1901 & 307
\end{tabular}

$355 \quad 1902 \quad 815$

\begin{tabular}{l|ll}
773 & 1904 & 668
\end{tabular}

\begin{tabular}{l|l}
149 & 1917 \\
482
\end{tabular}

$470 \quad 1926 \quad 726$

416 I927f. I

572 I 93 I $\quad 628$

\begin{tabular}{l|l}
447 & 1936 \\
257 & $\mathbf{7 2 5}$
\end{tabular}

\begin{tabular}{l|l}
257 & 1937 \\
736 & 559
\end{tabular}

$\begin{array}{ll}736 & 1938 \\ 6 & 612\end{array}$

603 I939 686

356 I940 687

\begin{tabular}{l|ll}
514 & I 941 & 674
\end{tabular}

652 1943 I I 4

634 I945 365

4491948

27 I $949 \quad 316$

648 I950 $\quad 787$

646 I $969 \quad 467$

69 I $1970 \quad 548$

633 r982 791

\begin{tabular}{l|l}
598 & 1989 \\
5 & 247
\end{tabular}

524 I $990 \quad 825$

\begin{tabular}{l|l}
28 & I 991 \\
797 & 475
\end{tabular}

797 I993 680

$\begin{array}{lll}102 & 1994 & 205\end{array}$

$1031997 \quad 580$

$392005 \mathrm{a} \quad 40$

$76 \quad 2013 \quad 507$

$4772016 \quad 827$

754 2018c 542

$3172021 \quad 623$

$3912023 \quad \cdot 45$

$590 \quad 2025 \quad 360$

47 I $2030 \quad 450$

$4892040 \quad 424$

$8 1 8 \longdiv { 2 0 4 8 } \quad 6 2 2$

$6 8 3 \longdiv { 2 0 5 9 } \quad 5 7 3$ 


\begin{tabular}{|c|c|c|c|c|c|}
\hline CIL IV & & CIL IV & & CIL IV & \\
\hline 2060 & 706 & $2400 \mathrm{f}$ & 527 & $3152 a$ & 715 \\
\hline 2066 & 602 & $2400 \mathrm{~g}$ & 526 & 3169 & 415 \\
\hline 2069 & 819 & $241 \mathrm{I}$ & $5 \mathrm{I}$ & 3185 & 370 \\
\hline 2070 & 402 & 2411 a & 3 & 3199 & 595 \\
\hline 2082 & 490 & $2413 a$ & 479 & $3200 \mathrm{~g}$ & 325 \\
\hline 2083 & 77 & $24 \mathrm{I} 3 \mathrm{f}$ & 478 & 3202 & 655 \\
\hline 2089 & 387 & $24 \mathrm{I} 3 \mathrm{~h}$ & 547 & 3207 & $6 \mathrm{I}$ \\
\hline 2107 & II 5 & $2413 \mathrm{k}$ & $75^{2}$ & 3291 & 163 \\
\hline 2119 & 724 & 2414 & 608 & 3294 & 161 \\
\hline 2124 & 92 & 2416 & 694 & 3347 & 118 \\
\hline $21+5$ & 204 & 2425 & 654 & 3356 & $4^{8}$ \\
\hline 2146 & 727 & $243^{\circ}$ & 302 & 3377 & 322 \\
\hline 2152 & 563 & 2437 & 301 & 3388 & 771 \\
\hline 2155 & 46 & 2440 & 452 & 3403 & I 74 \\
\hline 2157 & 210 & $244^{8}$ & 388 & 3406 & 389 \\
\hline 2163 & $54 \mathrm{I}$ & $245^{\circ}$ & 453 & 3407 & 821 \\
\hline 2168 & 353 & 2455 & 422 & 3409 & 182 \\
\hline 2183 & 299 & 2457 & 493 & 3433 & I 33 \\
\hline 2188 & 619 & 2459 & 200 & $344^{2}$ & 722 \\
\hline 2193 & 469 & 2460 & $8 \mathrm{I}$ & $347 \mathrm{I}$ & 128 \\
\hline 2202 & 454 & 2464 & 303 & 3485 & 175 \\
\hline 2204 & 664 & 2476 & 286 & 3494 & 639 \\
\hline 2210 & 810 & 2479 & 330 & 3502 & 178 \\
\hline $224 I$ & 613 & 2483 & 29 & 3508 & 4 \\
\hline 2246 & 614 & 2495 & 350 & $35 \mathrm{I2f}$ & 669 \\
\hline 2254 & $43 \mathrm{I}$ & 2498 & 329 & 3525 & 97 \\
\hline 2256 & 326 & 2507 & 748 & 3529 & 147 \\
\hline 2258 & 619 & 2508 & 260 & 3539 & 198 \\
\hline $2258 a$ & 494 & 2513 & 371 & 3572 & I 48 \\
\hline 2288 & 620 & 2514 & 55 & 3579 & 570 \\
\hline 2292 & $6 ; 8$ & 2517 & 57 & 3580 & $35 \mathrm{I}$ \\
\hline 2308 & $4 \mathrm{II}$ & $2520 \mathrm{a}$ & 59 & 3674 & 163 \\
\hline $2310 \mathrm{~b}$ & $45^{8}$ & 2524 & 58 & 3678 & 161 \\
\hline $2310 \mathrm{k}$ & $80 \mathrm{I}$ & $254 \mathrm{I}$ & 75 & 3687 & I89 \\
\hline $23 \mathrm{II}$ & 776 & 2544 & 73 & 3695 & 19 \\
\hline 23191 & 665 & 2887 & 201 & 3702 & $13^{2}$ \\
\hline 2325 & 413 & 2953 & 488 & 3710 & 544 \\
\hline 2326 & 293 & 2975 & 142 & 3711 & 609 \\
\hline $233 \mathrm{I}$ & 53 & $2993 \mathrm{t}$ & 176 & 3718 & 183 \\
\hline 2353 & 525 & $2993 y$ & 90 & 3722 & 47 \\
\hline 2360 & 582 & 2995 & I 4 & 3726 & 95 \\
\hline 2374 & 630 & $306 \mathrm{I}$ & 673 & 3760 & 150 \\
\hline $23^{80}$ & 78 & 3072 & 783 & 3774 & 23 \\
\hline 2387 & $27 \mathrm{I}$ & 3083 & $55^{6}$ & 3775 & $15^{2}$ \\
\hline 2393 & 620 & 3092 & 765 & 3784 & 518 \\
\hline 2396 & 753 & 3117 & $75 \mathrm{I}$ & 3789 & 256 \\
\hline 2398 & 280 & 3 I $2 \mathrm{I}$ & 750 & 3796 & 799 \\
\hline 2399 & 766 & 3123 & 82 & 3804 & 347 \\
\hline 2400 & 501 & 3129 & 743 & 3811 & 723 \\
\hline $2400 \mathrm{a}$ & 822 & 3135 & 781 & $3^{822}$ & $9 \mathrm{I}$ \\
\hline $2400 \mathrm{~d}$ & $5^{29}$ & 3136 & $812 a$ & $3^{828}$ & 127 \\
\hline $2400 \mathrm{e}$ & 528 & 3 I 49 & 806 & 3832 & $696 a$ \\
\hline
\end{tabular}


CIL IV

3864

3867

3872

3874

$388 \mathrm{I}$

3882

3884

3885

3888

3889

3890

$389 \mathrm{I}$

3905

3909

3935

$394^{8}$

3950

3957

3964

3976

3990

3992

4000

4001

4007

4016

4023

4024

$404^{2}$

4050

$405 \mathrm{I}$

4053

4057

4074

4078

4080

4089

409 I

4098

4100

4106

4107

4110

4112

4114

4118

4120

4122

4126

4133

4138

4140
CIL IV

$433 \quad 4$ I 47

3 I 4150

I92 4I6I

1934169

$240 \quad 4182$

$24 \mathrm{I} 4 \mathrm{I} 84$

2424185

5174186

7294189

$796 \quad 4196$

$434 \quad 4^{207}$

$305 \quad 4225$

$483 \lcm{4227}$

$718 \quad 4235$

$613 \quad 4239$

$636 \quad 4244$

2824257

3634262

$423 \quad 4268$

$539 \quad 4270$

6424273

2I I 4276

$395 \quad 4277$

$662 \quad 4278$

3I 4280

I $8 \quad 4286$

$460 \lcm{4287}$

$456 \quad 4288$

$4 9 \longdiv { 4 2 9 4 }$

$5 4 \longdiv { 4 2 9 5 }$

$354 \quad 4297$

$757 \quad 4299$

$332 \quad 4302$

4I9 4304

$820 \quad 4308$

82943 IO

85 43 I I

$593 \quad 4327$

$408 \lcm{4334}$

$523 \quad 4342$

5354345

$764 \quad 4353$

3694355

7214356

8I $2437^{\circ}$

$532 \quad 4378$

$543 \quad 4379$

3284380

$626 \quad 4382$

$807 \quad 43^{84}$

$24 \quad 4385$

3234398
CIL IV

\begin{tabular}{l|l}
86 & 4404
\end{tabular}

4624409

552 44 I I

544 I 8

I 104420

$557 \quad 4422$

6594429

4994430

I I 4439

\begin{tabular}{ll|l}
663 & 4447
\end{tabular}

524456

$755 \quad 4474$

$400 \quad 4477$

$677 \quad 4485$

$505 \quad 4488$

533 449I

$734 \quad 4495$

$564 \quad 4496$

2 I 4498

3624508

4144509

$637 \quad 45$ I 4

42 I 4519

$720 \quad 45^{28}$

$265 \quad 4533$

$273455^{\circ}$

$248455 \mathrm{I}$

2544562

$250 \quad 4563$

$267 \quad 4592$

$255 \quad 4596$

$279 \quad 4599$

25 I 4600

6 II 4602

2724603

$206 \quad 4610$

2084615

2684618

$266 \quad 462$ I

$2744622 \mathrm{~d}$

$275 \quad 4636$

$277466 \mathrm{I}$

2904668

$276 \quad 467$ I

3134690

2524699

$270 \quad 4704$

2954707

$288 \quad 47$ I 3

$253 \quad 47$ I 4

2874721

\begin{tabular}{l|l|l}
459 & 4723
\end{tabular}
42

794

536

I 16

269

393

320

487

464

540

$67 \mathrm{I}$

$3^{8} 4$

575

5 I I

685

786

$44^{\circ}$

43

610

732

584

324

607

430

I 13

497

$34^{\circ}$

749

368

455

538

386

746

648

4I

407

367

213

327

214

264

420

756

$74 \mathrm{I}$

466

650

342

566

745

373

372

714 


\begin{tabular}{|c|c|c|}
\hline CIL & & CIL IV \\
\hline 4753 & $5^{22}$ & 5007 \\
\hline $475^{8}$ & 717 & 5009 \\
\hline 4760 & 352 & 5011 \\
\hline 4764 & 643 & 5012 \\
\hline 4765 & 645 & 5020 \\
\hline 4767 & 3 I 4 & 5029 \\
\hline 4768 & 730 & $503 I$ \\
\hline 4772 & $3^{81}$ & 5035 \\
\hline 4776 & 644 & 5037 \\
\hline 4777 & 742 & 5065 \\
\hline 4783 & 506 & 5068 \\
\hline 4788 & 498 & 5069 \\
\hline 4793 & 366 & 5080 \\
\hline $48 \mathrm{II}$ & 392 & $508 \mathrm{I}$ \\
\hline 4814 & 89 & 5086 \\
\hline 4816 & 621 & 5087 \\
\hline $483 I$ & 731 & 5092 \\
\hline 4832 & 793 & 5094 \\
\hline 4833 & 647 & 5105 \\
\hline 4836 & $3^{83}$ & 5112 \\
\hline 4839 & 606 & $5+23$ \\
\hline 4855 & $8 \mathrm{I} 4$ & 5125 \\
\hline 4858 & 710 & 5127 \\
\hline $486 \mathrm{I}$ & 605 & 5130 \\
\hline 4862 & 648 & 5138 \\
\hline 4870 & 249 & 5150 \\
\hline 4874 & $55 \mathrm{I}$ & 5153 \\
\hline 4876 & 568 & 5158 \\
\hline 4887 & 777 & 5174 \\
\hline 4888 & 396 & 5181 \\
\hline 4889 & 397 & 5182 \\
\hline 4901 & 778 & 5184 \\
\hline 4903 & 337 & 5189 \\
\hline 4905 & 292 & 5195 \\
\hline 4906 & 401 & 5198 \\
\hline 4919 & 824 & 5202 \\
\hline 4946 & 406 & $5^{203}$ \\
\hline 4949 & 9 & 5213 \\
\hline 4950 & 775 & 5214 \\
\hline 4954 & 502 & 5225 \\
\hline 4957 & 702 & 5232 \\
\hline 4966 & 585 & 5233 \\
\hline 4967 & 586 & 5242 \\
\hline $497 \mathrm{I}$ & 587 & 5244 \\
\hline 4976 & 693 & 5246 \\
\hline 4977 & 613 & $525 \mathrm{I}$ \\
\hline 4983 & 769 & 5254 \\
\hline 4986 & 640 & 5266 \\
\hline 4987 & 739 & 5267 \\
\hline 4995 & 5 OI a & 5268 \\
\hline 4999 & 162 & 5274 \\
\hline 5006 & $75^{8}$ & 5275 \\
\hline
\end{tabular}

\begin{tabular}{l|ll} 
& CIL IV & \\
790 & 5279 & 830 \\
574 & 5280 & 760 \\
826 & 5296 & 599 \\
795 & 5298 & 52
\end{tabular}

$784: 5299 \quad 719$

$7595300 \quad 341$

$48 \mathrm{I} 5303 \quad 808$

$676: 5306 \quad 258$

5 I6 $534 I$

\begin{tabular}{l|l}
32 & 5347 \\
3 & 359
\end{tabular}

$38015352 \quad$ I95

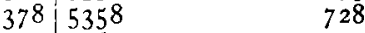

$357 \lcm{5360} \quad 740$

$8 7 \longdiv { 5 3 6 3 } \quad 4 0 9$

$747 \quad 5370$

$6 8 8 \longdiv { 5 3 7 1 }$

$58 \mathrm{I} 5372$

$4 8 5 \longdiv { 5 3 7 3 }$

46 I 5375

8025380

$43^{8} \quad 5386$

$2 2 \longdiv { 5 3 9 5 }$

$457 \quad 5398$

$3 7 6 \longdiv { 5 3 9 9 }$

$5 7 7 \longdiv { 5 4 0 6 }$

$377 \lcm{5408}$

$3 7 9 \longdiv { 5 4 I 3 }$

33 I 5417

$500: 5424$

$446 \quad 5430$

$448: 5432$

\begin{tabular}{l|l|l} 
I I I & $544^{8}$
\end{tabular}

344 5450

792 545 1

$554 \quad 5454$

$3 2 \longdiv { 5 4 5 7 }$

465 546 I

65 I 5466

I04 5476

701 5486

\begin{tabular}{l|l}
735 & 5488
\end{tabular}

2895494

$700 \quad 5499$

$695: 5504$

394 660 I

68 I 662 I

$699 \quad 6624$

$553 \mid 6626$

50Ib 6635

\begin{tabular}{|l|l|l}
553 & 664 I
\end{tabular}

$534 \mid 6672$

\begin{tabular}{ll|l}
259 & 6682
\end{tabular} 
CIL IV

6687

6697

6608

6716

6731

6733

6757

$676 \mathrm{I}$

6764

6768

6773

6779

6785

6796

6815

$68 \mathrm{I} 7$

6820

6825

6828

6838

6839

6842

6847

6849

6853

6864

6865

6868

6873

6877

6882

6884

6885

6887

6890

6892

6893

6894

6902

6905

6907

6909

7014

7037

7038

7059

7065

7069

7080

7086

7089

7090

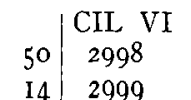

809 300I

398 3004

$768 \quad 3008$

$772 \quad 3009$

2963010

8163028

$561 \quad 3033$

8173038

2913050

$33 \quad 3052$

$385 \quad 3053$

I 443056

\begin{tabular}{l|l}
35 & 3057 \\
98 & 3060
\end{tabular}

\begin{tabular}{r|r}
737 & 3060 \\
635 & 3065
\end{tabular}

6353066

\begin{tabular}{l|l}
79 & 3067 \\
88 & 3069
\end{tabular}

\begin{tabular}{l|l}
88 & 3069
\end{tabular}

$338 \quad 3072$

$30 \quad 3074$

$343 \quad 3075$

$412 \quad 3076$

\begin{tabular}{l|l}
767 & 3079
\end{tabular}

I3 308 I

47329791

$63829848 \mathrm{~b}$

$434 \mathrm{a} 33^{893} \mathrm{~b}$

442 CIL X 4483

38 Ephem. epigr. VIII

$744 \quad 337$

$672 \mathrm{CE}$

$4^{\circ} 432$

3 I5 33

$596 \quad 35$

$80 \quad 37$

$546 \quad 38$

\begin{tabular}{r|r}
203 & 40 \\
68 & $4 I$
\end{tabular}

\begin{tabular}{l|l}
68 & $4 I$ \\
72 &
\end{tabular}

\begin{tabular}{l|l}
60 & 42
\end{tabular}

\begin{tabular}{l|l|l}
196 & 43
\end{tabular}

318

$698 \quad 44$

$375 \quad 45$

\begin{tabular}{l|l}
185 & 47
\end{tabular}

$445 \quad 48$

$738 \quad 49$

$604 \quad 50$

$6675 \mathrm{I}$

780.230
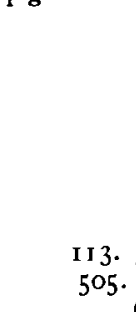

\section{3 .}

505.

8 II. 8
$8 \mathrm{r} 3$.

\begin{tabular}{l|ll}
230 & $\mathrm{CE}$ & \\
$23 \mathrm{I}$ & 686 \\
$2 \mathrm{I}$ & 232 & $5 \mathrm{IO}$
\end{tabular}

$223 \quad 233$

224

226

332

$235 \quad 333$

$238 \quad 349$

$234 \quad 35^{\circ}$

$236 \quad 35 \mathrm{I}$

$233 \quad 353$

$225 \quad 354$

$228 \quad 355$

$220 \quad 356$

229359

$216 \quad 926$

$222 \quad 927$

2I8 928

$215 \quad 929$

$237 \quad 93^{\circ}$

2I 93 I

$239 \quad 932$

$56 \quad 933$

$23 \mathrm{I} \quad 934$

227 935, If

$22 \mathrm{I} 935, \mathrm{I}_{4} \mathrm{ff}$

232936

$436 \quad 937$

$696 \quad 939$

$565 \quad 94^{\circ}$

$508 \quad 942$

703

943

31. 263

583. 828

669

704

675

212

677

317

785

$68 \mathbf{I}$

627

$5^{84.679 .808}$

482

304

I 4

671

636

34

702

$64 \mathrm{I}$

585

586

587

803

588

589

590

$59 \mathrm{I}$

592

593

\begin{tabular}{l|l}
476 & 945 \\
946
\end{tabular}

782.947

$594 \mathrm{f}$

27

597

598

819

$429 \quad 950$

I 44952

599

567

600

601

$6 \mathrm{I}_{4} \mathrm{f}$

602

668

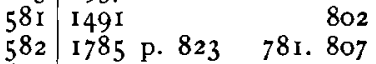

689 I 785 P. 824 574. 603 .

$674 \quad 673.680 .688$

685 I 785 p. 825

800

449. 471.690 I789

6991798

796

692

691 1810

616 
De 234

I319

2176

2177

2609

2610

26I I

$4 \mathrm{I} 8 \mathrm{I} \mathrm{b}$

5138

$5 \mathrm{I} 42 \mathrm{a}$

$5 \mathrm{I} 42 \mathrm{~b}$

$5142 \mathrm{e}$

$5142 \mathrm{~d}$

5143

5144

5145

5146

5147

5181 a

5723

6034

6035

6036

6326

6364

\begin{tabular}{r|l} 
& De \\
95 & $6398 \mathrm{a}$ \\
II & $6398 \mathrm{~b}$ \\
227 & $6398 \mathrm{c}$ \\
230 & $6398 \mathrm{~d}$ \\
$2 \mathrm{I} 4 \mathrm{~b}$ & 6400 \\
$2 \mathrm{I} 4 \mathrm{c}$ & $640 \mathrm{I}$ \\
$2 \mathrm{I} 4 \mathrm{a}$ & 6402 \\
46 & 6403 \\
263 & $6404 \mathrm{a}$ \\
274 & $6404 \mathrm{e}$ \\
275 & 6405 \\
277 & $6406 \mathrm{a}$ \\
276 & $6407 \mathrm{a}$ \\
243 & $6408 \mathrm{~b}$ \\
244 & 6409 \\
242 & 6410 \\
$24 \mathrm{I}$ & $64 \mathrm{II} \mathrm{a}$ \\
247 & $6412 \mathrm{c}$ \\
$3 \mathrm{I} 2$ & $64 \mathrm{I} 2 \mathrm{e}$ \\
435 & $6413 \mathrm{~b}$ \\
436 & 6414 \\
437 & $6418 \mathrm{~b}$ \\
439 & $64 \mathrm{I} 8 \mathrm{c}$ \\
563 & $6418 \mathrm{~d}$ \\
472 & $6419 \mathrm{e}$
\end{tabular}

\begin{tabular}{|c|c|}
\hline 2 I & 64 Igf \\
\hline & $6422 \mathrm{~b}$ \\
\hline & 6423 \\
\hline & 6424 \\
\hline & 6424 \\
\hline & $64^{2} 5$ \\
\hline & 6428 \\
\hline & 643 \\
\hline & 6433 \\
\hline 34 & 6434 \\
\hline 32 & 6435 \\
\hline & 6438 \\
\hline & $643^{8}$ \\
\hline & 6439 \\
\hline 152 & $644^{\circ}$ \\
\hline 129 & 6440 \\
\hline 40 & 6441 \\
\hline & $644^{2}$ \\
\hline & 6443 \\
\hline & 6443 \\
\hline & 6443 \\
\hline & 6444 \\
\hline 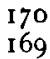 & \\
\hline & \\
\hline
\end{tabular}

171

I 43

I 81

J 82

183

I 84

I 86

I 89

I 57

I 56

I97

I 37

I $3^{8}$

200

$44^{6}$

729

$53^{2}$

525

297

298

299

97

I 92

Frommannsche Hofbuchdruckerei (Hermann Pohle) in Jena. - 3710 